\title{
Mito, spettacolo e società: Il teatro di Carlo Gozzi e il femminismo misogino della sua Turandot
}

Alla Commedia dell'Arte Carlo Gozzi si rifece, almeno inizialmente, perché gli offriva uno spettacolo da contrastare al teatro di Goldoni, di Chiari e, più tardi, al nuovo dramma borghese e alla comédie larmoyante. A questo scopo l'Improvvisa poteva offrirgli aiuti preziosi: la teatralità pura degli intrecci, svincolati da ogni rapporto con la vita reale e contemporanea; le Maschere, cioè tipizzazioni astratte; il buffonesco delle trovate comiche che, insieme alle maschere, escludeva ogni possibile idealizzazione delle classi inferiori, degradate a oggetto di comico; e, infine, il carattere nazionale della Commedia, che escludeva qualsiasi intrusione forestiera.

Ma anche se Gozzi si erge a strenuo difensore della Commedia dell'Arte, è chiaro che le sue fiabe hanno in realtà ben poco a che fare con l'Improvvisa e il loro tono, sempre dominato da personaggi seri, è ben più drammatico, mentre le varie trame non sono ricavate dalla solita tradizione scenica ma da racconti italiani ed orientali, nel loro adattamento teatrale parigino della Foire. L'esotismo e la magia, inoltre, erano anch'essi elementi ignoti alla Commedia dell'Arte e la loro fusione con la continua polemica letteraria sono chiara indicazione di come il commediografo fosse ben altro che un mero restauratore di scenari improvvisati.

Trionfano nelle fiabe i valori tradizionali, dall' amor coniugale ( $R e$ Cervo, $T u$ randot, Donna serpente e Mostro Turchino), all'amore fraterno, la fedeltà e la virtù della semplicità (Corvo, Pitocchi fortunati e Zeim re de' Geni). Nel Re Cervo, Pitocchi e Zeim vengono anche puniti i ministri traditori, mentre i lussuriosi sono sconfitti nella Zobeide e nel Mostro turchino. Gli ingrati, infine, vengono rimproverati duramente nell'Augellin Belverde. ${ }^{1}$

Per educare teatralmente, Gozzi predilige l'exemplum astorico e universale, che egli ricerca nella condizione atemporale della fiaba, in una forma di narrativa popolare, anche questa in opposizione ai sofismi ed alle complicazioni razionalistiche del periodo.

Il mondo evanescente, illusorio, folle delle fiabe gozziane non è certo il prodotto di una fantasia sbrigliata ed ingenua, ma il frutto di una tenace ricerca del "meraviglioso" e della sua immaginosa realizzazione scenica. Ci sono infatti studi come quello di Zorzi che hanno dimostrato come sia possibile applicare il ben 
noto schema variabile messo a punto da Vladimir Jakovlenic Propp alle fiabe del Gozzi, riscontrando nella loro trama gran parte delle funzioni o parties che il folklorista russo indica come elementi invariabili della composizione della fiaba.

Non mancano naturalmente nelle fiabe debolezze e difetti, anche d'origine teatrale, come una certa "frammentarietà" identificata anche da Starobinsky, ${ }^{2}$ ma è alla teatralità più ovvia che punta soprattutto Gozzi, deciso com'era prima di tutto a vincere i suoi avversari Chiari e Goldoni, sottraendo loro il pubblico. A tale scopo egli sottomette la sua fiaba a un'esigenza primaria di tensione e di ritmo che si traduce in azione scenica serrata. La suspense teatrale, uno degli artefizi più comunemente impiegati nelle fiabe gozziane, mantiene sempre sospeso l'animo dello spettatore, fino allo scioglimento finale.

Fin dalla nascita dell'idea stessa di teatro, la "corsa agli ostacoli" dei due amanti, contrastati da eventi, genitori e problemi di ogni tipo, corsa generalmente coronata dalla felice conclusione finale del matrimonio, è sempre stata uno degli argomenti preferiti sulla scena. Nel caso delle fiabe gozziane, la battaglia fra i sessi è usata sia per la suspense narrativa e scenica, che per illustrare la polemica morale e moralistica del commediografo.

La questione del femminismo nel Settecento è molto complessa ed indagarla a fondo trascenderebbe gli scopi di questo articolo. Ma è importante sottolineare che se il Settecento viene considerato, soprattutto in Francia, il secolo della donna, ${ }^{3}$ la società tuttavia non cessa di essere androcentrica: all'uomo appartenevano tutti i diritti. Sorta però la necessità di cercare una forma di collaborazione tra i sessi, moralisti e filosofi del tempo cercano di definire le caratteristiche intellettuali e morali delle loro interlocutrici e l'educazione diventa il punto focale di queste conversazioni. Sono quesiti che aprono preziose prospettive sull'idea di come la donna dovrebbe essere e sull'estensione delle conoscenze di cui è necessario dotarla, sempre purché il "gentil sesso" si munisca soprattutto di quel genere di cultura superficiale che rende piacevole e piccante la conversazione nei salotti con l'altro sesso. 4

E quindi anche in chiave pedagogica che va interpretato il conflitto tra i due sessi che domina il teatro di Carlo Gozzi il quale, pur non essendosi direttamente interessato della questione dell'educazione femminile come altri illuministi "femministi" italiani a lui ben noti (dal fratello Gasparo, all'abate Chiari, a Pietro Verri), ${ }^{5}$ frequentava regolarmente il salotto della cognata, la poetessa Luisa Bergalli Gozzi, forse la più nota femminista italiana del periodo. Il terrore ed il fantasma della "femme trop savante" erano quindi argomento che gli era certamente ben noto.

Nelle sue fiabe Gozzi crea ruoli femminili che, vedremo, spesso si oppongono all'etica normativa. Così la donna indipendente che vuole rifiutare il matrimonio a cui viene costretta, per esempio, viene da lui usata come un ottimo esempio di elemento socialmente sovversivo che egli intende correggere e raddrizzare, per mezzo dell' exemplum astorico presentato dalla fiaba. L'antagonismo scenico delle fiabe è spesso illustrato dalla lotta fra i due sessi, con uomini e donne i quali, 
benché nemici naturali, hanno però disperatamente bisogno gli uni delle altre. Per una quantità di ragioni sia sociali, che psicologiche o etiche, le donne del Gozzi rappresentano il fattore forse più fragile nella difesa della società contro la corruzione morale. Gli uomini "deboli", altrettanto colpevoli, permettono alle donne di diventare "insubordinate", di sfuggire cioè alla proprio "giusta" posizione sociale. Le donne di Gozzi rappresentano così una minaccia veramente seria contro il mantenimento dell'ordine sociale.

Se nelle sue Memorie egli poteva fare ben poco oltre all'osservare e deplorare questa trasformazione della società evidenziata dall'"insubordinazione" femminile, nelle fiabe tale problema viene risolto con completa soddisfazione del commediografo. Alla conclusione di ogni fiaba teatrale, dopo un susseguirsi di scene piene di suspense, dove l'insubordinazione femminile e il generale disordine sociale regnano indisturbati, Gozzi ricostituisce sempre l'ordine per mezzo della repressione finale conclusiva, una repressione ben poco problematica e che poteva far tirare un sospiro di sollievo al pubblico tradizionalista, maschile e femminile, seduto in teatro.

Se in alcune fiabe, come L'Amore delle tre melarance (dove Celio e la Fata Morgana sono nemici giurati per tutto lo sviluppo della commedia) o La donna serpente, la lotta tra i sessi deve essere combattuta fino all'ultimo sangue, fino alla morte cioè della protagonista, in Turandot è l'indipendenza morale del personaggio che viene invece annientata (con piena adesione, vedremo, della protagonista stessa, Turandot). Abbiamo così un duello filosofico, non fisico, più adatto ad una fiaba dalla polemica meno pesante, che riesce in tal modo a creare un personaggio femminile complesso ed ambivalente. Ne La donna serpente, invece, con i suoi straordinari effetti scenici, osserviamo ben più apertamente $i$ conflitti coniugali (consci e subconsci), affiancati da quelli familiari e sociali, con Farruscad, il marito di Cherestanì (la donna serpente), "uomo incostante, donna molle più ch'uom", il quale esemplifica il totale rovesciamento del ruolo sessuale/coniugale codificato dall'ordine tradizionale patriarcale, auspicato dal commediografo.

L'ideologia gozziana, in fondo, riflette semplicemente la posizione tipica della sua classe di nobile decaduto, sprovvisto di potere sia economico che finanziario. E un'aristocrazia che cerca di aggrapparsi a vecchi valori tradizionali che avevano portato successo e potere negli anni passati. E un'aristocrazia quindi anche decisa a rifiutarsi a qualunque vero cambiamento sociale, che possa recare serie trasformazioni sia in quel microcosmo sociale che è la famiglia, sia nel macrocosmo della società.

$* * * * * * * * * * *$

Ricco di complessità e particolarmente riuscito è l'esempio di sovversione sociale offerto dalla principessa cinese Turandot che "nell'abborrimento estremo ch'ha del sesso maschil . . morrebbe prima d'assoggettarsi a . . uomo superbo, pria d'esser moglie" (2.5). 
Con Turandot, Gozzi si allontana decisamente dalla caricatura della donna diabolica, che aveva invece preferito per altri personaggi femminili. Il duello che Turandot combatterà col principe Calaf sarà quindi un duello intellettuale e amoroso, mentre l'intelligentissima eroina era riuscita ad influenzare abilmente le leggi del suo paese, per potere eliminare tutti coloro che minacciavano ciò che essa considerava il suo onore più alto, l'indipendenza. Il femminismo a oltranza di Turandot e la sua indipendenza giurata costituiscono quindi un attacco minaccioso contro l'ordine patriarcale e familiare, attacco tanto piu pericoloso in quanto l'eroina regale è dotata di bellezza sublime, intelligenza acutissima e cultura immensa.

Per eliminare la minaccia contro l'ordine sociale causata dal comportamento di Turandot, abbiamo già notato, la principessa verrà completamente distrutta non come personaggio in sé, ma come valore metaforico. Verrà così messa in atto una reintegrazione dell'ordine codificato patriarcale, che nella scena conclusiva domerà completamente l'essenza stessa del personaggio di Turandot. Ė una ristrutturazione gerarchica rinvenibile anche nella scelta linguistica dei segni che definiscono la protagonista di questa fiaba. Turandot è una "bisbetica domata" la cui sconfitta va codificata e formalizzata anche nel linguaggio perché, nell'ambiguità affascinante del personaggio, essa rappresenta un'antagonista dotata di un'umanità e fascino troppo potenti e pericolosi.

La fonte della fiaba di Turandot era derivata dalla "Histoire du Prince Calaf et de la Princesse de la Chine" nella raccolta di Les milles et un jour: Contes Persanes, nell'edizione francese di Le cabinet des Fées. Conveniamo però con Bosisio che le vicende della principessa irraggiungibile, bella e crudele, ha tradizioni ben piu antiche, e si trova integralmente anche nel poema Haft Paikar (Le quattro bellezze), del poeta persiano Nizâmi. Il nome stesso della principessa, del resto, è persiano (TùrânDokht), e significa la fanciulla del Turan, toponimo impiegato in Persia per indicare la Russia asiatica e la Cina. È possibile che a Gozzi fosse nota anche La princesse de Carizme, commedia esotica di Alain René Lesage, ispirata anch'essa alla medesima fonte remota. ${ }^{7}$

Turandot esemplifica particolarmente bene anche quella speciale proiezione inconscia sia positiva che, come in questo caso, negativa della psiche maschile, definita Anima dallo psicologo svizzero Carl J. Jung:

A still more subtle manifestation of a negative Anima appears in some fairy tales in the form of a princess who asks her suitors to answer a series of riddles or, perhaps, to hide themselves under her nose. If they cannot give the answers, or if she can find them, they must die - and she invariably wins. The Anima in this guise involves men in a destructive intellectual game. We can notice the effect of this Anima trick in all those neurotic pseudointellectual dialogues that inhibit a man from getting into direct touch with life and its real decisions. He reflects about life so much that he cannot live it and loses all his spontaneity and outgoing feeling. (Franz 179) 
Anche nel ciclo eroico enucleato e sviluppato da Joseph Campbell nel suo $\mathrm{He}$ ro with a Thousand Faces (1. Alienazione dell'eroe; 2. Richiamo dell'Avventura; 3. Apoteosi e Ritorno) figurano diversi esempi di eroi (ed eroine) che si rifiutano al ciclo eroico, soffocando e bloccandone una delle tre fasi. Nel caso di Turandot, l'eroina rifiuta la seconda fase, cioè l'Avventura, e tale rifiuto, spiega Campbell, nei miti viene spesso metaforizzato attraverso il rifiuto all'unione cosmica o al matrimonio. In un altro racconto orientale, del principe Kamar al-Zamam e della principessa Budur, abbiamo addirittura due protagonisti che preferiscono torture e sofferenze indicibili pur di riuscire a non sposarsi (65-68).

Il rifiuto all'Avventura e al matrimonio viene spesso simbolizzato anche nel sonno senza possibilità di risveglio della principessa vergine, che pud̀ venire svegliata soltanto dal bacio del suo principe. Da Biancaneve alla Bella addormentata nel bosco, gli esempi di sonno verginale sono innumerevoli. Il castello dove tutti gli abitanti giacciono addormentati, rappresenta il più profondo e pericoloso degli abissi, dove si annega la coscienza individuale e si dissolve e disperde l'energia indifferenziata. Se non si raggiunge la fase psicologicamente vittoriosa dell'individuazione, simboleggiata dall'arrivo del principe/eroe che sveglierà col suo bacio non solo la principessa ma anche tutta la sua corte, il ciclo eroico viene interrotto e l'energia psichica rimarrà per sempre indifferenziata. Questo non è certo quanto avviene nella fiaba di Turandot, e ricordiamo come nella versione della fiaba musicata da Puccini, il Principe Calaf, con la sua nota romanza "Nessun dorma", fosse addirittura riuscito a sconfiggere anche la minaccia stessa del sonno mitico perpetuo (Adami e Simoni 3.1).

La Bella Addormentata, un personaggio, abbiamo visto, assai comune nelle fiabe folkloristiche, è, proprio come Turandot, figura affascinante quanto pericolosa. Se da un lato essa rappresenta col suo sonno l'apoteosi della bellezza e l'appagamento di ogni desiderio maschile di perfezione e completezza (cioè la madre totale o la sorella, amante, sposa sublime), dall'altro raccoglie anche l'altra proiezione maschile materna, quella che si era sviluppata negativamente o in repressione. Tale proiezione materna negativa (che comprende sia la madre assente ed inarrivabile che quella castratrice o edipicamente proibita), promette invece soltanto annientamento e distruzione, proprio come la Medusa, che trasformava gli uomini in pietra, se guardata direttamente, senza l'aiuto di uno specchio (Campbell 110-11). Di nuovo troviamo i medesimi topoi anche in questa fiaba di Gozzi, perché il ritratto di Turandot, una volta guardato dai principi "stolti o incauti" li seduce ed ipnotizza immediatamente, impietrendoli se non fisicamente, almeno psicologicamente.

Il teatro di Gozzi si rifa alle fiabe folkloristiche anche per quanto riguarda la mancanza di prospettiva spaziale e temporale. I personaggi gozziani sono sempre vecchi o giovani a priori; il tempo per loro non trascorre, né è scandito dall'alternarsi di giomo e notte. I personaggi della fiaba (popolare e gozziana) sono figure senza corpo, a cui mancano i riferimenti di un mondo interiore e di un ambiente circostante. Rappresentano così proiezioni psichico/mitiche che esi- 
stono nell'assenza di ogni spessore corporeo e spirituale. Non sono in alcun modo vincolati all'ambiente: dalla presenza assolutamente incongrua di maschere veneziane nella Cina di Turandot, alla ripetizione irrazionale ed automatica di un Truffaldino che taglia le melarance una dopo l'altra, senza neanche domandarsene il perché.

**********

La "ridicola fola persiana" di Turandot, come Gozzi stesso la definisce nella prefazione, scelta, almeno consciamente, per farne una fiaba "senza malie e senza trasformazioni" (226) (visto che i rivali avevano ascritto il suo successo precedente soprattutto alle fantasmagorie del $\operatorname{Re}($ ervo), contiene, come abbiamo visto, una quantità di temi sotterranei mitici, che ne assicurarono l'immenso successo, immediato e futuro, prima in Germania e poi nel resto d'Europa. Sempre nella prefazione, Gozzi aggiunge:

Non morì dopo la sua nascita, questa favolosa opera scenica. Ella si recita tuttavia ogni anno, con quel buon esito, ch'è la sola cagione della collera dei suoi fiabeschi nimici. (226)

Il processo con cui l'indipendenza antisociale di Turandot viene progressivamente diminuita e, infine, distrutta è segnalato fin dalla prefazione della fiaba, nella scelta linguistica gozziana delle didascalie. Si tratta infatti di una "ridicola fola . . . [basata] su tre indovinelli e due nomi ... che [non] sono veramente una gran base per... tener tre ore fermo e legato ad una serietà, tanto discorde coll'argomento, un uditorio colto" (225-26). L'intento del narratario appare quindi inizialmente polemico, e la scelta del racconto orientale viene presentata come un episodio casuale, senza nessuna importanza. Ma ci sono due elementi che Gozzi stesso non può fare a meno di rilevare, sempre nella prefazione: l'abilissima suspense ("ottimamente rilevata dai perspicaci" [226]), ed il fatto che anche se si trattava di una "ridicola fola" era pur sempre una "favolosa opera scenica" di grande successo. Notiamo quindi l'intento polemico apertamente espresso in primo piano e, ricordata a malapena e quasi di sfuggita (ma ricordata comunque), la consumata abilità dell'autore, il che dimostra come il commediografo fosse comunque sempre ben conscio della propria arte.

Il sipario si apre sullo sfondo della porta della città di Pechino sopra la quale ci sieno molte aste di ferro piantate; sopra queste si vedranno alcuni teschi fitti, rasi, col ciuffo alla turca (1.1). Turandot, continua a spiegare l'autore nelle didascalie, parla alteramente . . con sussiego e sempre in tuono accademico quando presenta i suoi indovinelli ( 2.4 , e altrove).

Quando Calaf comincia a mostrarsi vittorioso, si nota un rapido cambiamento, con la principessa che diventa sdegnosa, in seguito sdegnosissima e addirittura invasata (2.4) al momento della sconfitta. L'iniziale attrazione per Calaf l'aveva fatta alternare tra il sentirsi perplessa e risoluta (2.4). E questa un'indecisione che si svilupperà in seguito in tenerezza (5.2), e mentre tutti gli astanti star commossi 
e piangeranno durante la licenza finale, nel momento stesso in cui rifiuterà completamente l'orgogliosa indipendenza che aveva caratterizzato la struttura stessa del suo personaggio, Turandot si rivolgerà agli altri caratteri ed al pubblico parlando in atto riflessivo (5.2).

In una parallela decostruzione dei dialoghi dei vari personaggi, sempre in riferimento alla principessa cinese, notiamo molte similitudini con le didascalie, con dialoghi e didascalie che seguitano a scandire e puntualizzare il processo con cui viene distrutta l'essenza femminista del personaggio. L'aio di Calaf, Barach, durante l'iniziale fortunato incontro col suo principe, procede col descrivere immediatamente il "teatro abbominevol ... di crudeltà inaudite" che è diventata Pechino. La colpa, naturalmente, è di "Turandot, la figlia unica d'Altoum imperatore, bella quanto crudel . . cagion di barbarie, e lutti e lacrime" (1.1).

Quali sono le armi con cui questa "cruda" fanciulla si rifiuta di obbedire all'ordine codificato patriarcale e di accettare con serena acquiescenza lo sposo sceltole dal padre? Sempre secondo Barach le armi di Turandot sono "la bellezza inimitabile . . profonda perspicacia di mente e . . . [l'essere] sì avversa al sesso mascolin" (1.1). Le tre cose, accoppiate ai "molti ritratti per le corti in giro" della principessa, hanno creato questa situazione anarchica e caotica, piena di principi decapitati, e quindi chiaramente contraria all'ordine sociale, codificato e patriarcale.

Le parole di Barach contro Turandot diventano sempre pil dure. Trattandosi dell'aio di Calaf e quindi, mitologicamente, del padre putativo dell'eroe protagonista, Barach funge da perfetto portavoce dell'ordine tradizionale gerarchizzato, che Gozzi farà trionfare alla fine della fiaba. Turandot, racconta Barach, rifiuta i tentativi del padre di darle qualche "sposo di real stirpe" perché è "indomita superba" (1.1). Questa "vipera ... terribil donna ... tigre" ha avuto l'ispirazione dei tre indovinelli ("diabolica richiesta") per cui ora "l'inumana si pavoneggia" (1.1) del suo successo e della morte dei pretendenti. Allo stesso tempo, i padri dei giovani decapitati stanno tutti appressandosi per invadere la Cina e vendicare la morte dei propri figli. Il microcosmo caotico sovversivo del rifiuto al matrimonio di Turandot ha quindi finito per creare addirittura un macrocosmo caotico sovversivo di futura distruzione totale dell'intera nazione cinese.

La grande bellezza di Turandot, quella calamita che attrae innumerevoli giovani principi dagli stati confinanti, è anche potenziata dall'immagine che ne è stata fissata atemporalmente ed ipnoticamente in un ritratto. E un ritratto baciando il quale "volentier muoio" dice, prima di morire, l'ultimo decapitato (1.2). L'aio di lui Ismaele, invece, piange dirottamente e getta a terra e calpesta lo stesso ritratto, esclamando: "O maledetto, diabolico ritratto, qui rimanti" (1.2). Si tratta così di "un'immagine infernale" (1.4) con cui l'arte ha potuto potenziare la sovversione dei valori sociali operata dal personaggio della principessa ribelle, in carne e ossa. Se i segni linguistici puntualizzano il lato eroico/animalesco della principessa ("cruda, tigre, velenosa"), per il ritratto Gozzi si rifá invece al lessico oratorio sacerdotale, cristiano o pagano. Il ritratto di Turandot viene così de- 
scritto dagli altri personaggi non solo come "diabolico e infernale", ma anche come "faccia tremenda di Medusa . . . velenosa effigie . . . e mortifera peste" (1.3).

Nei suoi ammonimenti sempre più angosciati a Calaf (perché rifiuti l'avventura eroica ed eviti l'incontro con Turandot), Barach esorterà il principe a "mirare il teschio del principe infelice ancor fumante", mentre la didascalia sottolinea che un orrido carnefice chinese con braccia ignude e sanguinose . . . pianterà il capo del principe di Samarcanda su uno dei pali, fitti davanti alla porta di Pechino (1.3). E da notare che Barach, questo portavoce dell'ordine sociale tradizionale e codificato, non si mostra particolarmente generoso con nessuna altra donna, neanche con la propria moglie la quale, poveretta, non solo non aveva mai sovvertito proprio niente, ma lo aveva addirittura accolto e sposato senza che egli le avesse mai onestamente rivelato la propria vera identità. Ma Schirina ha avuto il torto di rallegrarsi con la figlia, una delle schiave favorite di Turandot, della vittoria di Calaf sui tre enigmi, così che anch'essa da "femmina incauta . . garrula ghiandaia" vittima della propria "stolta debolezza femminile" diventa, nelle parole del marito, "ingrata sposa" (non si capisce troppo bene ingrata di cosa), e addirittura "folle" (3.6). Nel momento supremo in cui gli schiavi di Turandot si appresseranno a Barach con dei bastoni per strappargli il segreto del nome di Calaf, tutti i pensieri di questo portavoce del tradizionalismo gozziano correranno immediatamente (e non troppo amorevolmente) alla moglie: "Paga sarai Schirina. Or t'è palese l'effetto del tuo errore" (4.1), in una totale deplorazione della piccola indiscrezione della moglie, ora innalzata ad azione diffamante ed orrenda, degna dei più tremendi sensi di colpa.

Il secondo portavoce dell'autorità codificata, il re Altoum, dimostra invece un virtuosismo linguistico più complesso, nel difficile ed ambivalente rapporto dialogico stabilito con la figlia ribelle, Turandot. Qui Gozzi deve giocare con un personaggio colto mentre cerca di districarsi dal consueto conflitto metastasiano tra amore e dovere. Il re Altoum è assoggettato ad una "barbara figlia, nata per mio tormento ... [ [che] non si spoglia di crudeltà". Ma nella tensione melodrammatica che ne consegue, viene anche delineata la solitudine completa del monarca, il quale "non ritrova mai chi doni consiglio in tanta doglia" (2.2).

La maestà dei personaggi regali della fiaba viene sempre diminuita e contrastata dal dialetto delle maschere. Gozzi usa il gemellaggio ereditato dall'Improvvisa sia per alleggerire il dramma che per atemporalizzarlo ed universalizzarlo. Ne deriva un contrasto un po' stridente tra personaggi completamente opposti, un contrasto però efficace nella sua comicità. Così, nella stessa scena, Pantalone interrompe Altoum: "Cara Maestà, no saveria che consegio darghe", ed anche lui si sente particolarmente turbato dal fatto che tutti questi principi "se inamora de un retrattin" (1.3), mentre Tartaglia suggerisce opportunamente di sacrificare, invece di cento cavalli al Cielo e cento porci alla Luna, "quella porchetta della principessa" (2.2).

La disperazione del re per avere a che fare con una figlia "perversa orgogliosa 
crudele vana ostinata cagione d'ogni mia angoscia e morte" (2.3), subito scimmiottata dal "gran maledette femene" di Brighella, si risolve agevolmente in immediata felicità, nell'ultima scena. "Ognun s'allegri" esclama Altoum quando l'ordine sociale si ristabilisce e ci si appresta alle nozze tra Calaf e Turandot, una figlia che viene adesso chiamata "mia cara figlia", mentre il padre esclama con gioia: "Io ti perdono tutto il duol che mi desti. In questo punto compensi al padre tuo tutte le offese" (5.2). Di nuovo la maschera (in questo caso Pantalone) commenta subito con la sua mimesi: "Nozze, nozze. Siori dottori, le daga logo", il che annuncia la vera e propria commemorazione dell'evento e la completa risoluzione della suspense di tutta l'azione scenica precedente.

Piuttosto facile da decostruirsi è invece la Turandot espressa dai segni linguistici di Calaf. Qui Gozzi prende in prestito il consueto vocabolario amoroso di origine cortese, che ben si adatta all'eroico protagonista innamorato. Con la mente Calaf indovinerà i tre enigmi, con l'amore darà a Turandot la possibilità di indovinare il suo nome e, infine, con la noncuranza propria dell'eroe mitico vincitore, riuscirà a trionfare dove ognuno aveva prima fallito. Calaf aveva sentito "alcune fole ... inventate dal volgo ignaro" su Turandot, ma non ci aveva fatto caso perché, dice, "i casi miei chiaman altro che amori" (1.1). Pure, col coraggio dell'incoscienza, elemento indispensabile per mettere in atto la potenzialità dell'eroismo mitico, il principe raccoglie il ritratto perche "baie son queste" e, da tipico eroe vittorioso, a Barach che gli intima di chiudere gli occhi, ribatte con una certa alterigia: "Eh via, stolto, m'offendi" (1.3).

Per descrivere l'innamoramento di Calaf, Gozzi si rifà ai lazzi della Commedia dell'Arte, e nelle didascalie spiega che il principe con lazzi sostenuti s'incanta con esso (il ritratto). Un'occhiata, e l'immagine viene immediatamente mutata linguisticamente in "dolce effigie". "Gli occhi benigni" [e le] care rosate guancie, amabili pupille, ridenti labbra" di Turandot lo conquistano ed ipnotizzano (1.3). L'eroe è automaticamente pronto per il duello erotico/mentale, mentre esclamerà per la prima volta il pluriripetuto ritornello: "Morte pretendo, o Turandotte in sposa" (2.3). Come per Altoum, anche le apostrofi troppo letterariamente serie e petrarchesche di Calaf (se per Altoum, Gozzi aveva attinto al patrimonio linguistico drammatico, per Calaf si è rifatto a quello lirico), vengono subito riequilibrate e diminuite dalla mimesi comica della maschera. Pantalone così lo ammonisce subito: "Ma, cara Altezza, e cara vita mia . . . non so che gusto, che abbiè a vegnirvi a far scannar, come un cavròn" (2.3).

Calaf loda in modo particolare "la bella voce, bella presenza, e raro spirto e insuperabil mente" di Turandot (2.5). A pensarci bene, è piuttosto tragico che proprio "quel raro spirto e insuperabil mente", i quali lo avevano conquistato, Turandot sacrificherà a Calaf, alla società cinese ed agli spettatori seduti in platea, nella conclusione della fiaba. In realtà, Gozzi vorrebbe sottolineare per il pubblico che la Turandot reazionaria della scena conclusiva, la fanciulla che domanda perdono in atto riflessivo (5.3) per i suoi errori passati, è ancora una donna intelligente, anche se cambiata. Vedremo, nella conclusione della nostra 
analisi, se il commediografo sia riuscito veramente in tale intento.

Nel corso della fiaba, il personaggio di Turandot si era sviluppato secondo tre distinte personalità parallele. Le prime due caratterizzazioni sembrano contraddittorie, la terza, invece, vorrebbe essere una sintesi dei due opposti precedenti, ma è una sintesi che non persuade troppo. Aiutata dalle didascalie e da un bravo scenografo, la prima Turandot entra sempre in scena velata, vestita riccamente alla chinese, con aria grave e baldanzosa (2.4). E questo un velo (in realtà assai poco cinese ma arabo) che la principessa toglie quando vuole ipnotizzare Calaf con la sua bellezza (e ci riesce). E un velo che essa lacera furiosa quando il principe riuscirà a sciogliere i tre enigmi. Questa Turandot baldanzosa e altera parla spesso della sua "gloria" e dell" "abborrimento estremo al sesso maschil" (2.5). La principessa è perfettamente consapevole che il cielo le dette "acutezza e talento" e cadrebbe morta nel divano "se fosse vinta d'alcuno" (2.5). Secondo questa prima Turandot "tutti perfidi gli uomini son ... fingono amore per ingannar fanciulle". Orgogliosamente, essa rifiuta d'esser "moglie soggetta ad uomo e . . . purché 'l stranier non vinca ... [ [farà] tutto" (3.2).

E questa una fase femminista e orgogliosa del personaggio che nel corso della fiaba si indebolirà sempre più sia perché alternata dalla titubanza sempre più preponderante della seconda personalità, che per il disegno strutturale della fiaba stessa. Dobbiamo infatti ricordare che il personaggio disegnato da Gozzi come confidente di questa Turandot orgogliosa ed avversa agli uomini è Adelma, che il pubblico già conosce come intenta a tradire la principessa, perché innamorata essa stessa di Calaf. Le ultime preghiere di Turandot: "Cielo, soccorri Adelma, ch'io possa svergognarlo e scacciarlo" (4.3), (parla naturalmente di Calaf), comunicano così agli spettatori seduti in platea l'ambivalenza completa del messaggio. Con "cielo" e "soccorri" Gozzi si è rifatto al lessico ecclesiastico oratorio, ma la struttura della richiesta stessa, fatta a chi le sta mentendo, ottiene la doppia funzione di indebolire e umanizzare la caratterizzazione teatrale di Turandot.

Parallelo alla degressione della Turandot indipendente ed autoritaria, Gozzi disegna così il progressivo rinforzarsi della Turandot innamorata. Dal "costui mi fa pietà" (2.5) espresso fin dagli inizi a Zulima, si procede alla "compassione" (2.5), per arrivare al "tormento di vederlo afflitto e piangente" (4.3). Non sorprende che ben presto Gozzi adotterà per questa seconda personalità di Turandot il lessico lirico della poesia d'amore petrarchesca, mentre la principessa finalmente ammetterà che "difender più non posso il mio cor da costui" (5.2).

Nella conclusione della fiaba, Gozzi eliminerà l'opposizione tra le due fasi contrastanti, fondendole in una terza personalità, un bravo portavoce dell'ordine sociale, oramai trionfalmente ristabilito. Ne risulta un personaggio teatralmente incomprensibile, che non ha più niente a che fare né con l'orgogliosa femminista, né con la tenera e titubante innamorata. Le ultime parole di Turandot non sono infatti né di rispetto filiale per il padre, né di amore incondizionato per Calaf e neppure di rabbia per Adelma, la sua migliore amica, che l'aveva tradita (tutti sentimenti che sarebbero stati logicamente e teatralmente comprensibili). Le ulti- 
me parole di Turandot sono invece rivolte al "gentil popolo de' maschi". La principessa non solo vuole che essi sappiano che, chi sa perché, or a essa "gli ama tutti", ma spera anche che "il gentil popolo de' maschi" mostri "qualche segno di perdon" al suo "pentimento" per "un abborrimento sì ostinato al sesso mascolino" che Turandot adesso giudica "folle" (5.2).

Si tratta di una classica licenza, un envoi di sapore quasi medioevale che non tralascia perfino la ripetizione di una corta sinopsi di tutti gli avvenimenti della fiaba stessa. È una conclusione che ha certamente confortato sia Gozzi che gli altri spettatori reazionari veneziani del tempo, e che può forse confortare ancora coloro che per la propria sicurezza sociale o psicologica hanno bisogno di rifarsi a valori patriarcali stabili e tradizionali. E una conclusione che ha però anche indebolito immensamente il personaggio principale della fiaba, trasformando questa gloriosa principessa, ribelle ed anticonformista, in un burattino che nell'ultima scena riassume meccanicamente l'azione appena rappresentata, chiedendo addirittura perdono per l'asse strutturale stesso su cui si era imperniata l'intera azione scenica della fiaba.

$* * * * * * * * * * *$

Non intendiamo qui tracciare la storia della fortuna della Turandot gozziana, da Goethe a Schiller, a Hoffmann, fino a Brecht, soltanto in Germania. Rimandiamo i lettori alle ottime introduzioni già citate di Petronio e di Bosisio, che documentano compiutamente il successo di Turandot in Italia e all'estero. Basti ricordare che sia tra i Romantici che nei tempi più recenti si è sempre rimasti particolarmente affascinati dal contrasto comico/serio della fiaba. E un contrasto che ha dato noia ad alcuni, mentre altri hanno visto nell'eterogeneità gozziana una sovversione non solo del realismo illuminista ma addirittura di tutta la struttura teatrale tradizionale stessa.

Si continua ad interpretare Gozzi ancora romanticamente, come il portavoce del mito e del sogno, e le fiabe, e non soltanto Turandot, si prestano particolarmente bene alle interpretazioni musicali. La propensione mitologica di questo autore settecentesco veneziano è stata così apprezzata da una quantità di compositori tra cui ricordiamo Wagner, Strauss, Prokofiev, Busoni e Puccini.

Nel tardo Ottocento Andrea Maffeio aveva ritradotto in Italiano la traduzione tedesca della Turandot di Schiller, che aveva messo particolare enfasi sul lato mitico della fiaba. Fu alla versione del Maffeio che s'ispirò il già ricordato libretto della Turandot, che Giuseppe Adami e Renato Simoni scrissero per Puccini. E questa l'ultima opera di Puccini che il maestro, morto nel 1924, lasciò incompiuta. ${ }^{8}$ E un'opera degli anni Venti, d'impronta romantico/verista e dalla scenografia sempre sontuosamente barocca, com'era stato del resto anche per la fiaba originale di Gozzi. Vogliamo concludere ricordando brevemente la versione pucciniana di questa favola, anche perché essa continua ad essere ancora molto popolare in Italia e all'estero, spesso addirittura l'unica versione con la quale la maggior parte degli spettatori contemporanei si siano mai avvicinati al favoloso perso- 
naggio di questa principessa cinese, anticonformista e ribelle.

L'eroina settecentesca di Gozzi si era rivelata personaggio ben più interessante della Turandot pucciniana, patologicamente frigida ed inumana. L'eredità romantica, ancora fortemente sentita nel libretto di Adami e Simoni, ha suggerito dei cambiamenti significativi, che riflettono soprattutto una scissione profonda fra le varie personalità della principessa cinese. L'aggettivo "pura", ripetuto continuamente a partire dall'inizio dell'opera ha sostituito il "cruda" di Gozzi, in una repressione sessuale che caratterizza lo spirito di questa femminista, tardo romantica. E un'inibizione che viene ampiamente compensata dall'orgia di sangue che nell'intepretazione operistica piuttosto morbosa del mito era sembrata necessaria ai due librettisti, per sostituire la suspense erotico/intellettuale gozziana con un diverso tipo di suspense. La crudelissima folla domanda sempre del carnefice, augurandosi "che la lama guizzi e sprizzi fuoco e sangue" (1.1).

Turandot "bianca al pari della giada, fredda come . . spada" si metamorfizza nella incorporea "Luna . . . amante smunta . . . esangue . . . taciturna . . . funereo lume" (1.1). I tre ministri, Ping, Pang e Pong, gli epigoni comici della Commedia dell'Arte, con un umorismo sempre più pesante e grossolano, descrivono intanto la vita di Pechino dove "si strozza! si sgozza! si trivella! si spella! si uncina e scapitozza! si sega e si sbudella!" (1.1). Per compensare alla nuova frigida purezza postromantica di Turandot, trionfa così il sadismo. Ne deriva uno strano equilibrio un po' morboso, che si mantiene per tutta l'opera. La musica, sentimentale e tematicamente ripetitiva, sostiene l'ipnosi mitica e misteriosa infusa nella fiaba dall'interpetazione romantica di Schiller, che ha ispirato l'opera. Lo sdoppiamento dei due personaggi, Turandot e Liù, in cui sono tradotte le due caratterizzazioni di orgoglio e tenerezza della Turandot gozziana, se può essere estremamente comprensibile da un punto di vista musicale (perché consente al compositore di alternare le arie da soprano drammatico di Turandot con quelle da soprano lirico di Liu), sfasa però completamente il personaggio della principessa cinese, che da ribelle si è qui fatta un misto di repressione sessuale e di sadismo. La principessa pucciniana è diventata infatti una vera aguzzina che tortura e fa torturare Liù fino alla morte ("Strappatele il segreto . . . alla tortura") (3.1). In Liù invece ("nulla sono ... una schiava, mio Signore"), che con dolcezza estatica spiega soavemente a Calaf che si è sacrificata "perché un dì, nella Reggia, m'hai sorriso" (1.1), abbiamo l'Agnello di Dio, la Donna/Angelo che, in un esempio di masochismo interpretato come dolcezza e umiltà femminili da questi librettisti postromantici, si ricollega facilmente all'immolazione per amore di tante figure femminili romantiche, dalla Gretchen goethiana alla Inez del Don Juan di Zorrilla. Sarà Liù che, nell'interpretazione pucciniana della favola, insegnerà per osmosi come amare a Turandot. E come imparerà ad amare Turandot, seguendo l'esempio di tale insegnante? Le didascalie descrivono la Turandot innamorata dell'ultima scena sconvolta, spaurita . . . con gli occhi velati di lagrime . . rabbrividendo . . quasi senza voce . . . con rassegnata dolcezza (3.5). Il personaggio regale ha così riassorbito in sé le qualità della schiava che si era immolata per 
amore, diventando figura un po' infantile, nevrotica quanto la principessa aguzzina, ma certamente meno spiacevole.

In un Cristianesimo tardo romantico e un po' morboso, che può ricordarci un Tommaseo o un Fogazzaro, nel dialogo finale dell'opera si effettua un'ultima scissione del personaggio di Turandot, uno sdoppiamento che completa la distruzione dell'affascinante e ambivalente principessa ribelle settecentesca, ideata da Carlo Gozzi. "La tua anima è in alto ma il tuo corpo è vicino" canta trionfalmente a Turandot il principe ignoto che era riuscito a conquistarla, mentre il coro degli astanti esalta l'arrivo dell'alba, apportatrice di Sole, di energia maschile e di amore e gioisce del tramonto ormai completo della vergine Luna/Turandot.

\section{SUNY at Stony Brook}

\section{NOTE}

1 Per una buona introduzione al teatro di Carlo Gozzi, vedi l'edizione delle Fiabe Teatrali a cura di Bosisio (11-71). Molto completa è anche l'introduzione alle Opere a cura di Petronio (9-46), anche se di orientamento un po' limitato in quanto principalmente marxista. Buone traduzioni inglesi recenti di alcune fiabe, anch'esse provviste di introduzioni interessanti che però spesso si rifanno a quelle già ricordate del Bosisio e soprattutto del Petronio, sono quelle edite da Di Gaetani e quelle a cura di Bermel ed Emery.

2 "C'est là définir un théâtre où tout est subordonné à l'exigence de l'action", nota Jean Starobinsky (435).

3 "La femme au dix-huitième siècle est le principe qui gouveme, la raison qui dirige, la voix qui commande" (De Goncourt 2.97. Citato in Bonatti 226).

4 "Siccome una volta le femmine solevano di rado uscire di casa e non v'avea conversazione comune fra uomini e donne, oggidi richiede l'usanza che spesso in compagnia si ritrovino e si facciano altri ragionamenti che di bucato, di telerie e d'altri affari appartenenti alle masserizie d'una famiglia" scrive Gasparo Gozzi (361). Sono stati pubblicati recentemente molti studi critici dedicati agli scritti femministi degli illuministi italiani, che si erano interessati dell'educazione femminile, ora che si cominciava a mitigare il rigido galateo spagnolo che aveva escluso le donne dal consorzio maschile e le aveva segregate in casa 0 in convento. Vedi, tra gli altri, l'estesa bibliografia degli articoli di Fido e di Bonatti. La citazione appena ricordata di Gasparo Gozzi è tolta dall'articolo della Bonatti (230).

5 Pietro Verri in particolare aveva dedicato alla figlia Teresa un intero Manoscritto da leggersi dalla mia cara figlia Teresa, scritto pochi mesi dopo la morte della moglie. Nel Manoscritto, tra l'altro, la esorta anche a leggere Locke e Rousseau per formarsi "un sistema che abbia per base la ragione, la sperienza, la umanita" per curare l'educazione dei propri futuri figli (197-98).

6 Per il testo della Turandot di Carlo Gozzi (prefazione e fiaba), ci siamo riferiti esclusivamente a quello gia ricordato, edito da Bosisio (225-316). Nel corso di questo articolo, per chiarezza, si useranno i corsivi per le didascalie e le virgolette per le parole pronunciate dai personaggi.

7 Per tutti questi dati cfr. l'introduzione di Bosisio a "Turandot: Fiaba chinese teatrale tragicomica" (223-24).

8 Poco prima della première del suo Trittico (dicembre 1918, al Metropolitan di New York), Puccini aveva già cominciato a ricercare il suo prossimo progetto operistico. Nel marzo dell'anno successivo, Renato Simoni gli aveva suggerito la Turandot di Gozzi, fiaba che era già stata musicata da Weber e Busoni. Entusiasta delle possibilità della fiaba, ancor piu 
nella versione di Schiller. Puccini ne commissionò il libretto a Renato Simoni e Giuseppe Adami. Il compositore cominció a dedicarsi alla partitura dell'opera nel gennaio del ' 21 , integrandovi autentiche melodie cinesi e avendo ricercato etnografie ed iconografie cinesi per suggerire l'immagine visuale dell'opera agli scenografi. Nel marzo del '24 Puccini aveva completato lo spartito fino alla morte di Liu. Non mancavano che i 15 minuti del duetto finale, quando il compositore mori per un cancro alla gola, nel novembre del ' 24 . Arturo Toscanini ingaggiò immediatamente Franco Alfano per finire l'opera, seguendo gli appunti di Puccini. Il rapporto fra i due fu, a dir poco, tempestoso, con Toscanini che alla première dell'opera alla Scala di Milano si rifiutò di proseguire oltre la morte di Liù, lasciando il podio ed annunciando: "Qui finisce l'opera, perché a questo punto il Maestro è morto". Per uno studio recente assai esteso sulla Turandot di Puccini, vedi Ashbrook and Powers. L'episodio Toscanini/Alfano appena citato è a pagina 152.

\section{OPERE CITATE}

Adami, Giuseppe, e Renato Simoni. Turandot. Dramma lirico in tre atti e cinque quadri. Musica di Giacomo Puccini. L'ultimo duetto e il finale dell'opera sono stati completati da F[ranco] Alfano. Milano .... : G. Ricordi e C. Editori, 1926.

Ashbrook, William, and Harold Powers. Puccini's "Turandot": The End of the Great Tradition. Princeton: Princeton UP, 1991.

Bonatti, Maria Ines. 'L'educazione femminile nel pensiero degli Illuministi e nei romanzi del Chiari". Annali d'Italianistica 7 (1989): 226-41.

Campbell, Joseph. The Hero with a Thousand Faces. 1949. Princeton: Princeton UP, 1973.

Fido, Franco. "Italian Contribution to the Eighteenth-Century Debate on Women". Annali d'lialianistica 7 (1989): 217-25.

Franz, Marie-Louise (von). "The Process of Individuation". Man and his Symbols. Ed. Carl G. Jung and Marie-Louise von Franz. New York: Doubleday, 1964.

Goncourt, Edmond et Jules (de). La Femme au dix-huitième siècle. Parigi: Flammarion, 1923.

Gozzi, Carlo. Fiabe Teatrali: Testo, introduzione e commento. Ed. Paolo Bosisio. Roma: Bulzoni, 1984.

Opere. Ed. Giuseppe Petronio. Milano: Rizzoli, 1962.

"The Love of Three Oranges", "Turandot", and "The Snake Lady" with a Bio-

Critical Introduction. Ed. and trans. John Louis Di Gaetani. New York: Greenwood Press, 1988. Five Tales for the Theater. Ed. and trans. Albert Bermel and Ted Emery. Chicago:

Chicago UP, 1989.

Memorie inutili. Ed. Domenico Bulferretti. 2 voll. Torino: UTET, 1928.

Gozzi, Gasparo. La Gazzetta veneta. Ed. Antonio Zardo. Firenze: Sansoni, 1915.

Propp, Vladimir Jakovlevic. Morfologia della fiaba. Torino: Einaudi, 1966. 1 ed. russa: 1928.

Starobinsky, Jean. "Ironie et mélancolie: Gozzi, Hoffmann, Kierkegaard". Sensibilità e razionalità nel Setrecento. Ed. Vittore Branca. Firenze: Sansoni, 1967. 423-62.

Verri, Pietro. “Manoscritto" per Teresa. Ed. Gennaro Barbarisi. Milano: Serra e Riva, 1983.

Zorzi, Luigi. "Struttura-fortuna della fiaba gozziana". Chigiana 31.11 (1976): 32-47. 